This is the Accepted Version of the article: Albert Van Eeckhout, Angel Lizana, Enric GarciaCaurel, Jose J. Gil, Adrià Sansa, Carla Rodríguez, Irene Estévez, Emilio González, Haolin Zhang, Juan C. Escalera, Razvigor Ossikovski, Ignacio Moreno, Juan Campos, "Indices of polarimetric purity to enhance the image quality in biophotonics applications," Proc. SPIE 10685, Biophotonics: Photonic Solutions for Better Health Care VI, 106850B (17 May 2018).

Available at DOI: https://doi.org/10.1117/12.2309375

(C) 2018 Society of Photo Optical Instrumentation Engineers (SPIE). One print or electronic copy may be made for personal use only. Systematic reproduction and distribution, duplication of any material in this publication for a fee or for commercial purposes, or modification of the contents of the publication are prohibited. 


\title{
Indices of polarimetric purity to enhance the image quality in biophotonics applications
}

\author{
Albert Van Eeckhout*a, Angel Lizana*a, Enric Garcia-Caurel ${ }^{\mathrm{b}}$, José J. Gil ${ }^{\mathrm{c}}$, Adrià Sansa ${ }^{\mathrm{a}}$, Carla \\ Rodríguez ${ }^{\mathrm{a}}$, Irene Estévez ${ }^{\mathrm{a}}$, Emilio González ${ }^{\mathrm{d}, \mathrm{e}}$, Haolin Zhang ${ }^{\mathrm{a}}$, Juan C. Escalera ${ }^{\mathrm{a}}$, Razvigor \\ Ossikovski $^{\mathrm{b}}$, Ignacio Moreno ${ }^{\mathrm{f}}$, Juan Campos ${ }^{\mathrm{a}}$ \\ ${ }^{a}$ Grup d’Òptica, Physics Department, Universitat Autònoma de Barcelona, Bellaterra, Spain; \\ ${ }^{b}$ LPICM, CNRS, École Polytechnique, Université Paris-Saclay, Palaiseau, France; \\ 'Laboratory of Polarimetry, ICE Universidad de Zaragoza, Zaragoza, Spain; \\ dDepartamento de Anatomía, Histología y Neurociencia, Universidad Autónoma de Madrid, Madrid, Spain; \\ eServicio de Anatomía Patológica, Hospital Universitario de Canarias, Santa Cruz de Tenerife, Spain; \\ fDept. de Ciencia de Materiales, Óptica y Tecnol. Electrónica, Universidad Miguel Hernández de Elche, Elche, Spain
}

\begin{abstract}
Recently, a set of polarimetric indicators, the Indices of Polarimetric Purity (IPPs), were described in the literature. These indicators allow synthesize depolarization content of samples, and provide further analysis of depolarizers than other existing polarimetric indicators. We demonstrate the potential of the IPPs as a criterion to characterize and classify depolarizing samples. In particular, the method is firstly analyzed through a series of basic polarization experiments, and we prove how differences in the depolarizing capability of samples, concealed from the commonly used depolarization index $P_{\Delta}$, are identified with the IPPs.

In the second part of this work, the method is experimentally highlighted by studying a rabbit leg ex-vivo sample. The obtained images of the ex-vivo sample illustrate how IPPs provide a significant enhancement in the image contrast of some biological tissues and, in some cases, present new information hidden in the usual polarimetric channels. Moreover, new physical interpretation of the sample can be derived from the IPPs which allow us to synthesize the depolarization behavior.

Finally, we also propose a pseudo-colored encoding of the IPPs information that provides an improved visualization of the samples. This last technique opens the possibility to highlight a specific tissue structure by properly adjusting the pseudo-colored formula.
\end{abstract}

Keywords: Depolarization, Biological tissue, Mueller matrix, Polarimetry, Imaging, Materials characterization.

\section{INTRODUCTION}

Polarization is a physical property of light which is related to the vibration direction of the electromagnetic waves. In light-matter interactions, this property can be modified by samples and the resultant changes encode the structural information of the investigated material. In particular, this phenomenology is widely studied in the polarimetry field, which encompasses a collection of measuring techniques and instrumentation that allow us (together with a given mathematical framework) to analyze and model the samples. The research and industrial interest in polarimetry are wide, being of prime importance in a wide number of applications [1-6].

One major application of the polarization based detection techniques is widely realized in biomedical imaging field thanks to its advantage demonstrated as non-invasive. Accordingly, polarimetric methods are nowadays used in biomedical applications, as for instance, for the study of skin diseases [7-9] or cancer early diagnosis [10-12].

The most used techniques for the characterization of biological samples can be indicated into two groups: polarization gating (PG) techniques and Mueller matrix (MM) measurements. PG methods allow the enhancement of the biological image contrast by properly selecting the polarization of the light illuminating the sample, and that of the polarization analyzer analyzing the light scattered by the sample. On the other hand, MM measurements, combined with certain associated analytical tools (mainly, MM decompositions), not only allow an enhancement of the image contrast of biological samples, but also permit a physical characterization of the studied samples and the observation of new 
structures. Although we can define two groups, PGs have been recently linked with MM methods, showing that PG can be generalized as particular cases of a discrete combination of MM coefficients [13].

Regarding the polarimetric analysis of samples, the polarization modification is directly related to the structural and chemical characteristics of the samples at different physical scales. In a general and complete description of the samples, three main polarimetric channels are considered: diattenuation, retardance, and depolarization, the two last being the most significant when dealing with biological tissues. What is more, these physical parameters can be derived from the Mueller matrix (MM) of the samples. The most common way to extract the information is to apply the Lu-Chipman decomposition, which decomposes the $\mathrm{MM}$ into three basic $\mathrm{MMs}$ (pure diattenuator $\mathrm{M}_{\mathrm{D}}$, pure retarder $\mathrm{M}_{\mathrm{R}}$, depolarizer $\mathrm{M}_{\Delta}$ ). From the pure retarder $\mathrm{M}_{\mathrm{R}}$, we can define different parameters with physical interpretation to characterize the retardance of the sample (e.g. global retardance, $\mathrm{R}$, linear retardance, $\mathrm{L}_{\mathrm{R}}$, neutral and extraordinary axes orientation, etc. [14-17]). Nevertheless, the depolarization study is restricted in the calculus of the depolarization power $(\Delta)$ or the degree of polarimetric purity $\left(\mathrm{P}_{\Delta}\right)$ which gives a value between 1 and 0 (from non-depolarizing to fully depolarizing elements, respectively).

Recently, a set of polarimetric indicators, the Indices of Polarimetric Purity (IPPs), composed by $\mathrm{P}_{1}, \mathrm{P}_{2}$, and $\mathrm{P}_{3}$ indices, were described in the literature [18-19]. These indicators allow to synthesize the depolarization content of samples, and provide further analysis of depolarizers (as it is the case of most biological tissues) than other existing polarimetric indicators. In addition, unlike other methods, the IPPs calculation does not require advanced algebraic formulation and results in three indicators of easy implementation.

In this work, we discuss the interests of using IPPs as the criterion to classify and characterize depolarizers. In Section 2, we theoretically present the numerical calculation method of the polarimertic purity indices from the measurement of the Muller Matrix. Afterwards, in Section 3, we describe the experimental set-up used to measure the Mueller matrix of the sample, it resulting in a well calibrated imaging Mueller Matrix polarimeter. In Section 4, we present a discussion related to the IPPs interpretation, through a series of six synthesized samples providing different IPPs values. Note that different IPP values are obtained in certain examples that give the same $\mathrm{P}_{\Delta}$, this leading to further synthetization of depolarizers. Next, in Section 5, we also prove the potential of the IPPs for biophotonics applications, and more specifically, in biological and biomedical imaging. In particular, the method is experimentally highlighted along Section 5 by investigating a section of a rabbit leg. A significant image contrast enhancement is obtained by using the IPPs based methods, compared to standard polarimetric images. Moreover, we show how some significant structures, which are hidden by using regular intensity images or standard polarimetric methods, are now contrasted when using the IPPs. This new information, computed by the IPPs, is better understood by considering their physical meaning: the IPPs does not quantify the degree of depolarization related to a MMs (as given by main used depolarizing indices), but rather synthesize the depolarizing behavior of a sample in three different channels related to different intrinsic depolarizing mechanisms. Finally, we also propose a pseudo-colored encoding of the IPPs information that leads to an improved visualization of samples. This last technique opens the possibility to adjust the tissue contrast by using customized pseudo-colored images. In this way, by properly constructing a figure of merit, based on IPPs, and tuning their respective weights, the visualization of a specific tissue structure in a sample is highlighted. A summary of the main conclusions extracted from the work are given in Section 6.

\section{INDICES OF POLARIMETRIC PURITY CALCULATION}

This section explains the main mathematical foundations into IPPs calculation from a measured Mueller matrix, defined previously by J. J. Gil [17-19]. We focus, not only in the calculation but also their relationship with well-known parameters as $\mathrm{P}_{\Delta}$ or a graphical representation of the IPP parameters.

Mueller matrices are $4 \times 4$ matrices, 16 coefficients in total, which has the information of the intensity, diattenuation, retardance, and depolarization. Firstly, we separate MM into different physically known elements:

$$
\hat{\boldsymbol{M}}=\boldsymbol{M} / m_{00}=\left(\begin{array}{cc}
1 & \boldsymbol{D}^{T} \\
\boldsymbol{P} & \boldsymbol{m}
\end{array}\right),
$$

where $\mathrm{m}_{00}$ is the mean intensity coefficient. $\mathbf{P}$ and $\mathbf{D}$ are called, respectively, the polarizance and diattenuation of $\mathbf{M}$. Note that two properties in $\mathbf{M}$ described as the intensity and the diattenuation can be identified, but we are not able to determine the retardance and the depolarization values of the matrix. In this case, we introduce an alternative technique, known as the Mueller decomposition, to analyze this retardance and depolarization. The most common decomposition used in the biological samples examination is the Lu-Chipman decomposition shown below [16] 


$$
\boldsymbol{M}=\boldsymbol{M}_{\Delta} \boldsymbol{M}_{R} \boldsymbol{M}_{D},
$$

in which the Mueller matrix $\mathbf{M}$ is decomposed into a depolarizer $\mathbf{M}_{\boldsymbol{\Delta}}$, a retarder $\mathbf{M}_{\mathbf{R}}$, and a diattenuator $\mathbf{M}_{\mathbf{D}}$.

Decompositions consist in subdivide matrices (i.e., $\mathbf{M}_{\Delta}, \mathbf{M}_{\mathbf{R}}$, or $\mathbf{M}_{\mathbf{D}}$ ) into a combination or a sequence of matrix factors with defined physical meanings. IPP calculus is based on Cloude's decomposition [17]. It decomposes M into four pure Mueller matrices with different weights as

$$
\boldsymbol{M}=m_{00} \sum_{i=1}^{4} \hat{\lambda}_{i} \hat{\boldsymbol{M}}_{J i},
$$

where $\mathbf{M}_{\mathrm{ji}}$ are the different pure Mueller matrices and $\lambda_{\mathrm{i}}$ are the eigenvalues of the associated covariance matrix $\mathbf{H}$ [14].The eigenvalues are restricted to the range $0 \leq \hat{\lambda}_{i} \leq 1$ and are ordered as $\lambda_{1} \geq \lambda_{2} \geq \lambda_{3} \geq \lambda_{4} \geq 0$. The eigenvalues are normalized by the sum of all of them, which is equal to $\mathrm{m}_{00}$. This relation between the different eigenvalues results in three completely independent values instead of the expected four. We define three dimensionless variables so-called indices of polarimetric purity (IPP) [17], as a combination of the normalized eigenvalues,

$$
P_{1} \equiv \hat{\lambda}_{1}-\hat{\lambda}_{2}, \quad P_{2} \equiv \hat{\lambda}_{1}+\hat{\lambda}_{2}-2 \hat{\lambda}_{3}, \quad P_{3} \equiv \hat{\lambda}_{1}+\hat{\lambda}_{2}+\hat{\lambda}_{3}-3 \hat{\lambda}_{4},
$$

Applying the eigenvalue restrictions, IPP are restrained by the following inequality:

$$
0 \leq P_{1} \leq P_{2} \leq P_{3} \leq 1
$$

We can relate IPP with a well-known parameter $\mathrm{P}_{\Delta}$

$$
P_{\Delta}^{2}=\frac{1}{3}\left(2 P_{1}^{2}+\frac{2}{3} P_{2}^{2}+\frac{1}{3} P_{3}^{2}\right)
$$

Using IPPs we can describe the samples in the same way as $\mathrm{P}_{\Delta}$. For instance, non-depolarizing systems are characterized by $\mathrm{P}_{\Delta}=\mathrm{P}_{1}=\mathrm{P}_{2}=\mathrm{P}_{3}=1$ and the total depolarizing matrices are described by $\mathrm{P}_{\Delta}=\mathrm{P}_{1}=\mathrm{P}_{2}=\mathrm{P}_{3}=0$. From the mathematically analysis of the totally depolarizing case, we conclude that pure depolarizers are composed by an equiprobable mixture of four orthogonal pure matrices. The advantage of the IPPs in retrospect of $\mathrm{P}_{\Delta}$ is the possibility of classifying depolarizers with different IPP but with the same $\mathrm{P}_{\Delta}$. [17].

Another tool to interpret the depolarizers is a 3D representation of IPPs, which is so-called the purity space (Fig. 1)

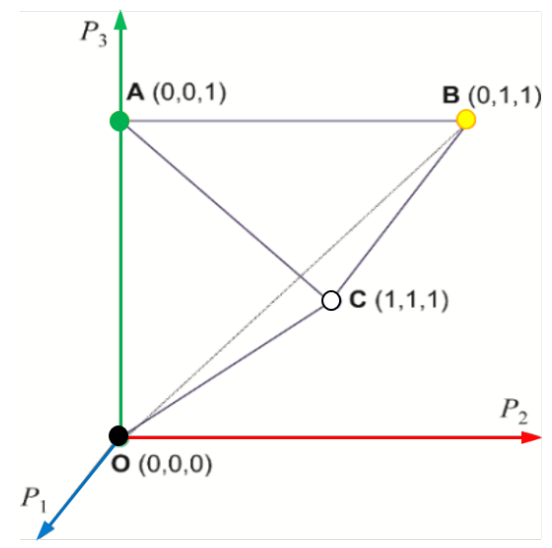

Figure. 1: Purity space representation comprised by the indices of polarimetric purity $\mathrm{P}_{1}, \mathrm{P}_{2}, \mathrm{P}_{3}$.

In this representation, the coordinate $\mathrm{O}(0,0,0)$ (black spot) corresponds to ideal depolarizers, whereas the coordinate $\mathrm{C}(1,1,1)$ (green spot) represents non-depolarizing materials. Moreover, we want to note that any other case can be represented in Fig. 1, with an intrinsic distance associated to an ideal depolarizer. In particular, any other case can be studied using its associated $\mathrm{P}_{1}, \mathrm{P}_{2}$, and $\mathrm{P}_{3}$. 


\section{EXPERIMENTAL SET-UP AND SAMPLES DESCRIPTION}

In this section, we describe the optical set-up implemented to measure the MM of different depolarizing samples, from which the IPPs indicators are latterly calculated. These IPPs are physically interpreted in the forthcoming Section 4, and used to improve the imaging of biological tissues in Section 5.

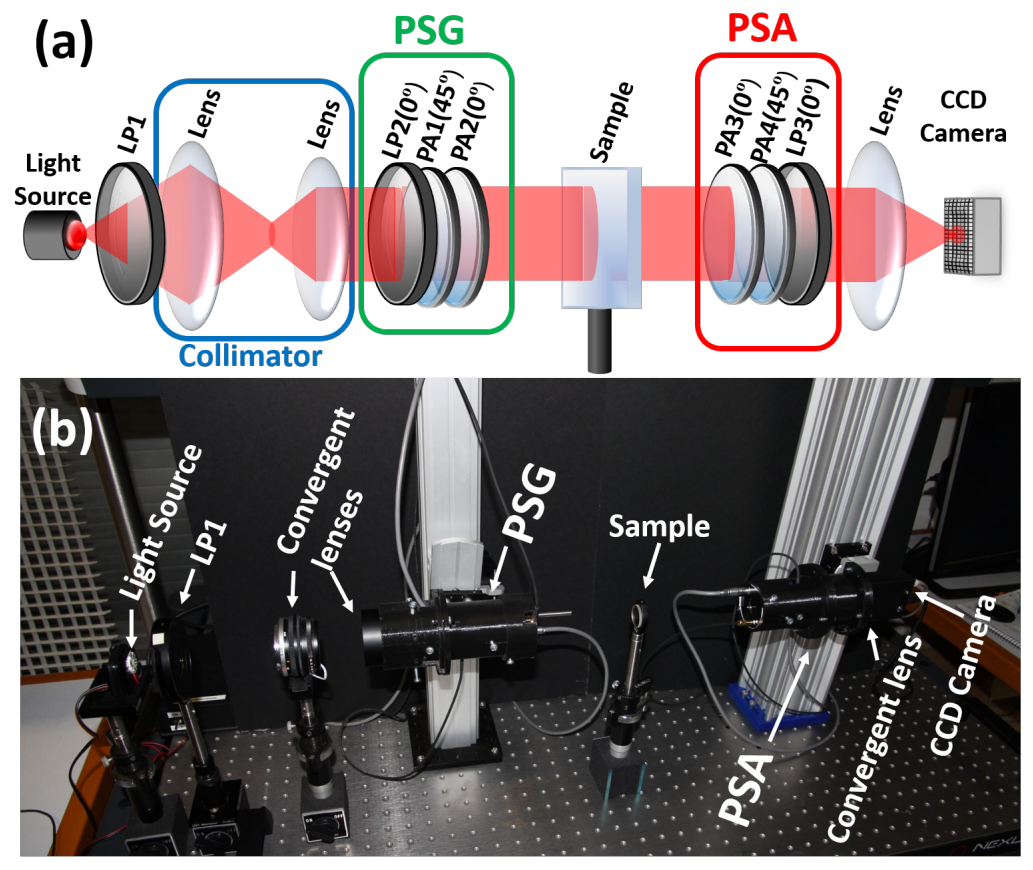

Figure. 2: Optical scheme of the complete imaging polarimeter used to measure the MMs of Samples.

The Mueller polarimeter used in the IPP analysis (shown in Fig. 2) is based on Parallel Aligned Liquid Crystal (PA-LC) retarders (four PA-LC Variable Retarders with Temperature Control; LVR-200-400-700- 1LTSC by Meadowlark Optics). The setup is basically composed of a collimated $627 \mathrm{~nm}$ light (Red LUXEON Rebel LED), a Polarization State Generator (PSG), Polarization State Analyzer (PSA) and a CCD Camera. First, the light is collimated by two convergent lenses (38 $\mathrm{mm}$ and $40 \mathrm{~mm}$ focal length, respectively) placed at the proper distances based on an afocal optical design. Afterwards, the collimated light is passing throughout a Polarization State Generator (PSG) (marked with a green rectangle in Fig. 2) which is able to generate any state of polarization by adjusting the retardances of the two PA-LC panels as discussed in [20]. Note that this PSG is composed by a linear polarizer (LP2) at $0^{\circ}$ degrees to the laboratory vertical reference axis (Glan-Thompson prism based polarizer by CASIX), a first PA-LC panel (PA1), oriented at $45^{\circ}$ to the laboratory vertical, and a second PA-LC panel oriented at $0^{\circ}$ (PA2). What is more, the polarized light illuminates the sample in transmission configuration with a controlled light power. This is achieved by adjusting the orientation of a linear polarizer (LP1) placed before the PSG.

The transmitted light is measured with a Polarization State Analyser (PSA) (marked with a red rectangle in Fig.2), which is constructed with the same optical elements than those in the PSG but in the inverse order. In this case, the linear polarizer LP3 is a dichroic sheet polarizer (by Meadowlark Optics). Finally, a convergent lens images the source on a CCD camera with certain magnification. As a lens, we use a TECHSPEC $®$ High-Resolution objective, distributed by Edmund Optics, with a focal length of $35 \mathrm{~mm}$. The camera is an Allied Vision Manta G-504B. It is a 5 Megapixel GigE Vision camera with the Sony ICX655 CCD sensor, with a $2452(\mathrm{H}) \times 2056(\mathrm{~V})$ resolution and a cell size of $3.45 \mu \mathrm{m} x$ $3.45 \mu \mathrm{m}$. The intention of this set-up, for the forthcoming study in Section 4, is the incoherent addition of all the responses produced by the different pure sample elements. For that reason, the image covers a large number of pixels on the CCD (area of $200 \times 200$ pixels), thus, effectively reducing the coherence area of the detector well beyond the spot size $[21]$.

In terms of efficiency, at least 16 images are needed to obtain the Mueller Matrix of the sample by applying the inverse method [14-16]. This MM is achieved in few seconds (0.4 to $2.2 \mathrm{~s})$, and the delay depends on the CCD exposure time. 


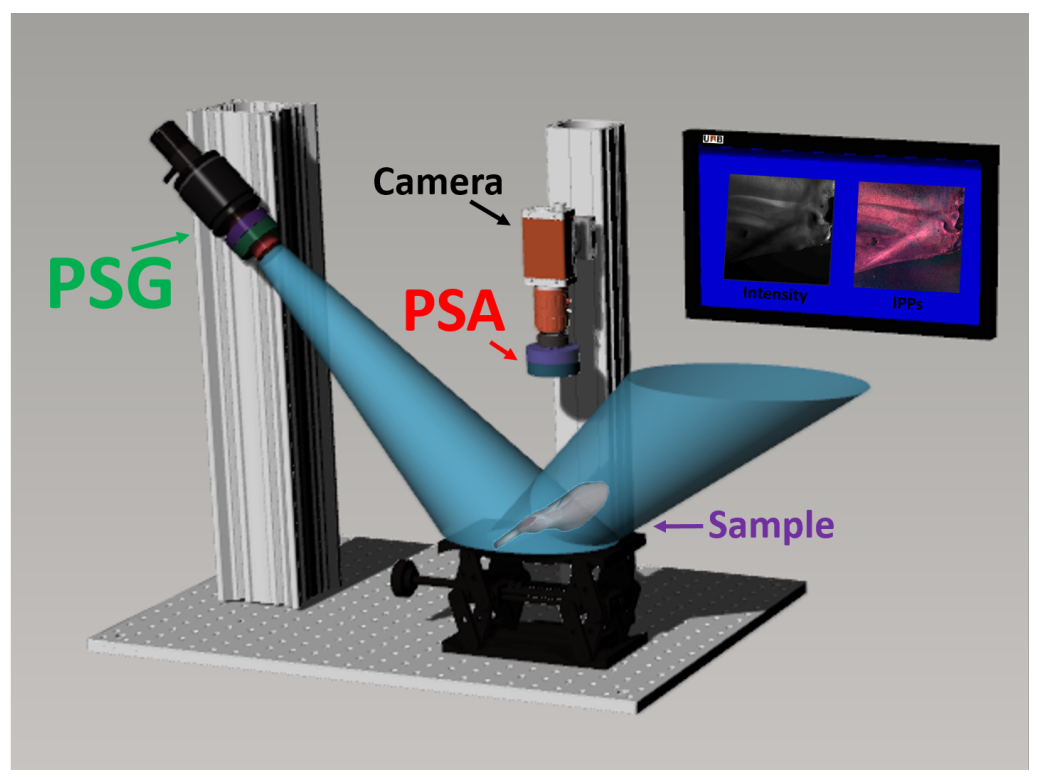

Figure. 3: Optical scheme of the complete image polarimeter used to measure the MMs of the ex-vivo sample.

The biological ex-vivo experiments in Section 5 are performed in reflection configuration because the nontransmissivity of the samples. Therefore, small variations in the setup are necessary for these experiments (Fig. 3). The essential variation is related to the light source. The illumination is now generated by a 4-Wavelength High-Power LED Source (Operated by DC4104 drivers distributed by Thorlabs) which is redirected by an optical fiber. We work with different wavelengths $(625 \mathrm{~nm}$ and $470 \mathrm{~nm})$ because the penetration length into the samples depends on the input wavelength [22] and it allows us to work with different depths. The other staged variation is the change on the focus distance of the objective in order to image the sample on the CCD. Finally, we obtain a resolution of $\sim 22 \mu \mathrm{m}$ and the region of interest are $2.2 \times 2.2 \mathrm{~cm}^{2}$.

Regarding the samples, they are divided into two groups: the non-biological samples (Section 4) and the biological samples (Section 5). The former group is basically composed of a combination of linear polarizers, quarter wave plates, and half-wave plates which will be presented with more detail in Section 4. Concerning the biological measurements, we perform a polarimetric study of an ex-vivo sample to highlight the improvement, in terms of image contrast, related to the use of the IPPs. According to a pathological analysis, the ex-vivo measured sample corresponds to a non-standardized dissection of a rabbit leg showing bone, striated muscle and connective tissue from tendons.

\section{EXPERIMENTAL EXPERIMENTS FOR THE ANALYSIS OF THE INDICES OF POLARIMETRIC PURITY}

The IPPs analysis is based on the construction and measurement of a set of depolarizing Mueller Matrices (MMs). These matrices are composed of an incoherent combination of pure (non-depolarizing) matrices such as linear polarizers or retarders. To construct different depolarizers, we model any depolarizer as the summation of different nondepolarizing matrices, with their respective weights, according to the following equation,

$$
\mathbf{M}=\alpha_{1} \mathbf{M}_{1}+\alpha_{2} \mathbf{M}_{2}+\alpha_{3} \mathbf{M}_{3}+\ldots
$$

where $M_{i}$ is the $M M$ of the $i$-th pure component $(i=1,2, \ldots)$, and the coefficients $\alpha_{i}$ give the weigh for each MM involved in the summation.

In the experimental case, the above-mentioned incoherent summation is performed by dividing the sample into different pure elements (Fig. 4), controlling the illumination of each element and spatially combining the information of the different sectors in the camera by means of a lens. Under this scenario, $\mathbf{M}_{\mathrm{i}}$ in Eq. (7) are defined by different pure elements (i.e. linear polarizer, quarter wave plate, half-wave plate, and air) spatially separated. In turn, the $\alpha_{i}$ are controlled by properly illuminating different elements. 

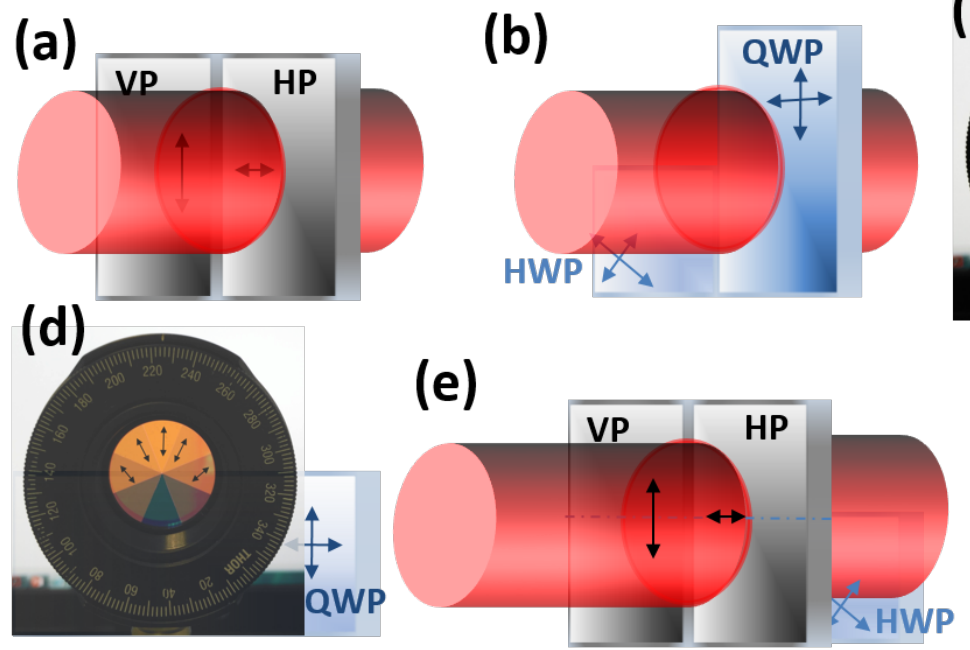

(b)
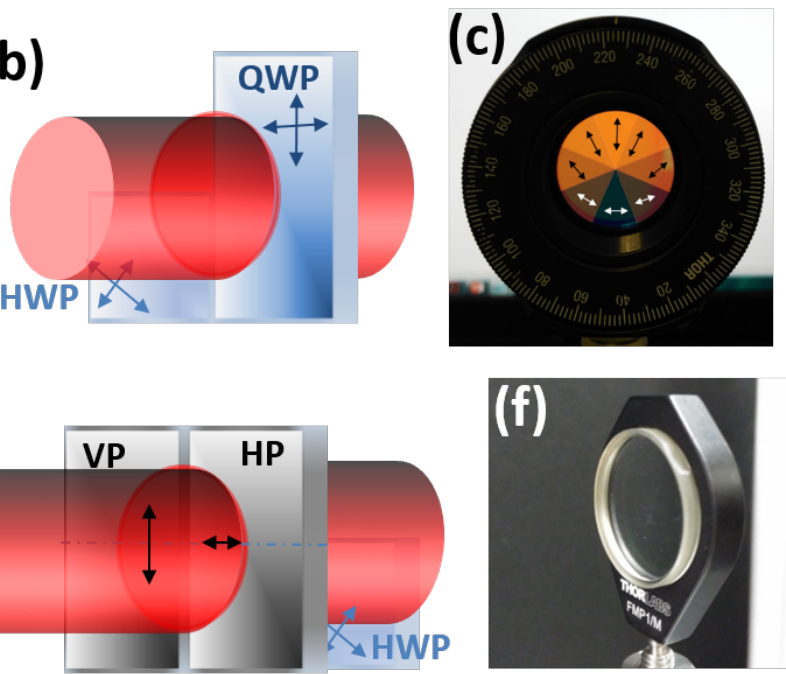

Figure. 4: Optical scheme for the samples: (a) "A"; (b) "B"; (c) “C”; (d) "D" and (e) "E"; and (f) picture of the sample "F".

Before starting the experimental analysis, let us summarize the possible scenarios under different combinations. In the case of a MM resulting in the combination of two pure matrices, $\mathrm{P}_{2}$ and $\mathrm{P}_{3}$ will always be equal to 1 , leaving only $\mathrm{P}_{1}$ adjustable in a range from 1 to 0 (varying weights and elements). The limit case when $\mathrm{P}_{1}$ equals to 0 corresponds to a combination of two orthogonal pure matrices with an equal weight. Following the previous argumentation, more than three pure element combinations are needed to obtain $\mathrm{P}_{2} \neq 1$ and more than four to obtain $\mathrm{P}_{3} \neq 1$.

In the following, we describe and analyze six different samples (labeled as " $A$ ", "B", "C", "D", "E" and " $F$ " in Fig. 4). Sample "A" consists of a combination of a vertical polarizer (VP) and a horizontal polarizer (HP) with the same illumination (Fig. 4(a)). The optical system $\mathbf{M}_{\mathrm{A}}$, using the Mueller formalism, can be described as,

$$
\mathbf{M}_{A}=\alpha_{1} \mathbf{M}_{H P}+\alpha_{2} \mathbf{M}_{V P}=\frac{1}{2} \frac{1}{2}\left(\begin{array}{llll}
1 & 1 & 0 & 0 \\
1 & 1 & 0 & 0 \\
0 & 0 & 0 & 0 \\
0 & 0 & 0 & 0
\end{array}\right)+\frac{1}{2} \frac{1}{2}\left(\begin{array}{cccc}
1 & -1 & 0 & 0 \\
-1 & 1 & 0 & 0 \\
0 & 0 & 0 & 0 \\
0 & 0 & 0 & 0
\end{array}\right)=\frac{1}{2}\left(\begin{array}{llll}
1 & 0 & 0 & 0 \\
0 & 1 & 0 & 0 \\
0 & 0 & 0 & 0 \\
0 & 0 & 0 & 0
\end{array}\right) .
$$

In this case, the weights $\alpha_{1}$ and $\alpha_{2}$ are both equal to 0.5 as the two different elements are equally illuminated. Note that $\mathbf{M}_{\mathrm{A}}$ is produced by the combination of two pure elements, and only presents two non-zero values placed at the matrix diagonal. Thus, we are implementing a 2D depolarizer [23]. The experimental and theoretical IPPs are calculated, accordingly to Eqs. 3 and 4, and presented in Table 1 . In this case, $\mathrm{P}_{2}$ and $\mathrm{P}_{3}$ equal to 1 as expected, and we obtain a P1 value equal to zero. We observe a slight disagreement in $\mathrm{P}_{1}$ between the experiment and the theory (i.e., 0 to 0.07 ). These small variations will be recurrent in this work as a consequence of the misalignments and the non-equally distributed light (the beam is not a perfect Gaussian distribution).

Table 1. Theoretical and experimental results for the different studied configurations.

\begin{tabular}{|c|c|c|c|c|c|c|c|c|}
\hline & \multicolumn{4}{|c|}{ Theory } & \multicolumn{4}{c|}{ Experiment } \\
\cline { 2 - 10 } & $\mathbf{P}_{\mathbf{1}}$ & $\mathbf{P}_{\mathbf{2}}$ & $\mathbf{P}_{\mathbf{3}}$ & $\mathbf{P}_{\Delta}$ & $\mathbf{P}_{\mathbf{1}}$ & $\mathbf{P}_{\mathbf{2}}$ & $\mathbf{P}_{\mathbf{3}}$ & $\mathbf{P}_{\Delta}$ \\
\hline $\mathbf{A}$ & 0.00 & 1.00 & 1.00 & 0.57 & 0.07 & 0.93 & 1.00 & 0.56 \\
\hline $\mathbf{B}$ & 0.40 & 0.71 & 1.00 & 0.57 & 0.40 & 0.70 & 1.00 & 0.59 \\
\hline $\mathbf{C}$ & 0.25 & 0.25 & 1.00 & 0.41 & 0.24 & 0.33 & 0.98 & 0.41 \\
\hline $\mathbf{E}$ & 0.00 & 0.00 & 0.00 & 0.00 & 0.05 & 0.19 & 0.25 & 0.13 \\
\hline $\mathbf{F}$ & - & - & - & - & 0.01 & 0.05 & 0.09 & 0.04 \\
\hline
\end{tabular}


Sample B consists of a combination of three different linear retarders with different weights $\alpha_{1}, \alpha_{2}$, and $\alpha_{3}$. As sketched in Fig. 4(b), half of the light is passing through a quarter wave-plate (QWP) with the fast axis oriented at $0^{\circ}$ (the orientations always refer to the vertical direction of the laboratory coordinate system). The rest half of the input power is also half divided itself, this leaving one-fourth of the total light illuminating a half wave-plate (HWP) with the fast axis oriented at $45^{\circ}$ and the other quarter directly passing through the air. The illuminations are equivalent to the weights being $\alpha_{1}=1 / 2, \alpha_{2}=1 / 4$ and $\alpha_{3}=1 / 4$.

$$
\begin{aligned}
& \mathbf{M}_{B}=\alpha_{1} \mathbf{M}_{Q W P}+\alpha_{2} \mathbf{M}_{H W P}+\alpha_{3} \mathbf{M}_{a i r}= \\
& \frac{1}{2}\left(\begin{array}{cccc}
1 & 0 & 0 & 0 \\
0 & 1 & 0 & 0 \\
0 & 0 & 0 & -1 \\
0 & 0 & 1 & 0
\end{array}\right)+\frac{1}{4}\left(\begin{array}{cccc}
1 & 0 & 0 & 0 \\
0 & -1 & 0 & 0 \\
0 & 0 & 1 & 0 \\
0 & 0 & 0 & -1
\end{array}\right)+\frac{1}{4}\left(\begin{array}{cccc}
1 & 0 & 0 & 0 \\
0 & 1 & 0 & 0 \\
0 & 0 & 1 & 0 \\
0 & 0 & 0 & 1
\end{array}\right)=\left(\begin{array}{cccc}
1 & 0 & 0 & 0 \\
0 & 1 / 2 & 0 & 0 \\
0 & 0 & 1 / 2 & -1 / 2 \\
0 & 0 & 1 / 2 & 0
\end{array}\right) .
\end{aligned}
$$

The non-zero values present in $\mathbf{M}_{\mathrm{B}}$, calculated in Eq. 9, are situated at the $3 \times 3$ right-bottom sub-matrix defined as $\mathbf{m}$ in Eq. 1 (block diagonal matrix). Therefore, the $\mathbf{M}_{\mathrm{B}}$ presents a combination of depolarization and retardance. In addition, $\mathrm{P}_{2}$ leads to be different from 1 as it is built from three pure MMs (i.e., Table 1).

The comparison between samples " $\mathrm{A}$ " and "B" demonstrates a significant interest because they have the "same" $\mathrm{P}_{\Delta}$ (Table 1), but their composition and the depolarizing response are not equal. Therefore, the different composition is discerned by IPP and depolarizers can be better characterized using this method.

The second group of examples ("C" and "D") illustrate how the combination of an ample number of pure matrices is not necessarily translated to the reduction of the IPP values. Presented in Fig. 4(c), case " $C$ " is the result of an incoherent summation of eight linear polarizers with different orientations (orientations are equally spaced in order to cover the principal directions which are marked by arrows in the Fig. 4(c)). The mathematical description of Mc, given in Eq. 10, presents how a 3D depolarizer [23] is synthesized.

$$
\mathbf{M}_{C}=\sum_{i=1}^{8} \alpha_{i} \mathbf{M}_{L P}\left(\theta_{i}\right)=\sum_{i=1}^{8} \frac{1}{8} \frac{1}{2}\left(\begin{array}{cccc}
1 & \cos \left(2 \theta_{i}\right) & \sin \left(2 \theta_{i}\right) & 0 \\
\cos \left(2 \theta_{i}\right) & \cos ^{2}\left(2 \theta_{i}\right) & \sin \left(2 \theta_{i}\right) \cos \left(2 \theta_{i}\right) & 0 \\
\sin \left(2 \theta_{i}\right) & \sin \left(2 \theta_{i}\right) \cos \left(2 \theta_{i}\right) & \sin ^{2}\left(2 \theta_{i}\right) & 0 \\
0 & 0 & 0 & 0
\end{array}\right)=\frac{1}{2}\left(\begin{array}{cccc}
1 & 0 & 0 & 0 \\
0 & 1 / 2 & 0 & 0 \\
0 & 0 & 1 / 2 & 0 \\
0 & 0 & 0 & 0
\end{array}\right),
$$

where $\mathbf{M}_{\mathrm{LP}}(\theta i)$ are the MMs of linear polarizers oriented to an angle of $\theta i$, the subscript $i$ denotes the specific polarizer sector $(i=1, \ldots, 8)$ with the same weight $\alpha_{i}=1 / 8$.

The arised $\mathrm{P}_{\Delta}$ and IPP values, presented in Table 1, differentiates sample " $\mathrm{C}$ " from the above studied ones. However, IPP are more explicit and describe the sample as a $3 \mathrm{D}$ depolarizer $\left(\mathrm{P}_{3}=1\right)$ contributing to a higher physical interpretation.

More complex samples can be described as in the case of sample "D" (Fig. 4(d)) which is similar to sample "C" but with the addition of a QWP. Note that half of light passing through the polarizers is illuminating a QWP (lower half) with its fast-axis oriented at $0^{\circ}$. The complexity of this case is increased by measuring the same construction but with different initial polarizer orientations (rotating the substrate observed in Fig. 4(d)). The theoretical and experimental IPP values obtained from the "D" sample are represented in Fig. 5 from different angular orientations.

By analyzing the results demonstrated in Fig. 5, a perfect fitting between theoretical and experimental results is realized. Specifically, $\mathrm{P}_{2}$ represents a stronger angle dependence among the three terms. Moreover, $\mathrm{P}_{2}$ and $\mathrm{P}_{3}$ are oscillating with a same tendency while $\mathrm{P}_{1}$ represents a contrary changing tendency compared to the other two terms $\left(\mathrm{P}_{2}\right.$ and $\mathrm{P}_{3}$ ) during the measurements. These results, when thinking in terms of image contrast, induce a calculation of differences between the IPP parameters $\mathrm{P}_{2}-\mathrm{P}_{1}$ and $\mathrm{P}_{3}-\mathrm{P}_{2}$, presented in previous works [19,23], to analyze the different depolarizing behaviors. 


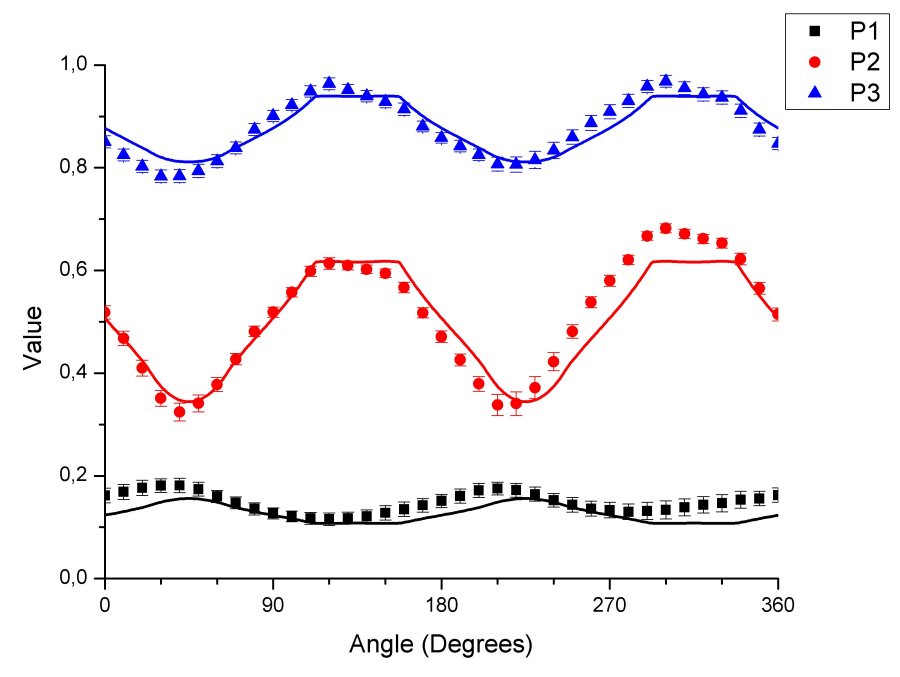

Figure. 5: Experimental and theoretical IPP values obtained for sample D. The three solid lines correspond to the theoretical responses and the symbols $\boldsymbol{\square}, \bullet, \boldsymbol{\Delta}$ represent $\mathrm{P}_{1}, \mathrm{P}_{2}$, and $\mathrm{P}_{3}$, respectively.

The last constructed sample (sample "E" sketched in Fig. 4(e)), is the synthesization of an ideal depolarizer (i.e., $P_{\Delta}=P_{1}=P_{2}=P_{3}=0$ ). The sample is divided into four regions, which can be described in two parts. First, the light illuminates equally an HP and a VP as illustrated in sample "A". Afterwards, half of each illumination in this construction, after passing throughout the polarizers, is modified by an HWP with the fast-axis oriented at $45^{\circ}$. $\mathbf{M}_{\mathrm{E}}$ can be mathematically described as,

$$
\begin{aligned}
& \mathbf{M}_{D}=\alpha_{1} \mathbf{M}_{V P}+\alpha_{2} \mathbf{M}_{H P}+\alpha_{3} \mathbf{M}_{H W P}\left(45^{\circ}\right) \mathbf{M}_{V P}+\alpha_{4} \mathbf{M}_{H W P}\left(45^{\circ}\right) \mathbf{M}_{H P}= \\
& \frac{1}{4} \frac{1}{2}\left(\begin{array}{llll}
1 & 1 & 0 & 0 \\
1 & 1 & 0 & 0 \\
0 & 0 & 0 & 0 \\
0 & 0 & 0 & 0
\end{array}\right)+\frac{1}{4} \frac{1}{2}\left(\begin{array}{cccc}
1 & -1 & 0 & 0 \\
-1 & 1 & 0 & 0 \\
0 & 0 & 0 & 0 \\
0 & 0 & 0 & 0
\end{array}\right)+\frac{1}{4} \frac{1}{2}\left(\begin{array}{cccc}
1 & 1 & 0 & 0 \\
-1 & -1 & 0 & 0 \\
0 & 0 & 0 & 0 \\
0 & 0 & 0 & 0
\end{array}\right)+\frac{1}{4} \frac{1}{2}\left(\begin{array}{cccc}
1 & -1 & 0 & 0 \\
1 & -1 & 0 & 0 \\
0 & 0 & 0 & 0 \\
0 & 0 & 0 & 0
\end{array}\right)=\frac{1}{2}\left(\begin{array}{cccc}
1 & 0 & 0 & 0 \\
0 & 0 & 0 & 0 \\
0 & 0 & 0 & 0 \\
0 & 0 & 0 & 0
\end{array}\right) .
\end{aligned}
$$

In Eq. $11, \alpha_{1}, \alpha_{2}, \alpha_{3}$ and $\alpha_{4}$ are all set to $1 / 4$ according to Fig. $4(\mathrm{e})$.

As observed in Eq. 11, an ideal depolarizer consist of a full-zero matrix except for the $\mathrm{m}_{00}$ term. In the ideal case, the depolarization studied values would be $P_{\Delta}=P_{1}=P_{2}=P_{3}=0$. However, in the experimental implementation, these values are slightly different from zero because of the misalignment and the beam shape disorder.

Finally, we performed an extra experiment (sample F) to compare the synthesized depolarizer with an ideally commercial depolarizer. In particular, the sample "F" is a DPU-25-A-Quartz-Wedge Achromatic Depolarizer distributed by Thorlabs (Fig. 4(f)). The experimental IPP values obtained are very close to zero (the ideal case). Note that the deviation of the experimental result to the theoretically one (i.e., zero) is introduced by the absence of misalignment errors. A comparison between the "E" and "F" experiments shows how by properly constructing samples from basic pure components, we can synthetize interesting materials, as the fabrication of pure depolarizers, in an easy and non-expensive way.

\section{BIOLOGICAL TISSUES IMAGE ENHANCEMENT BY USING THE INDICES OF POLARIMETRIC PURITY}

The investigation results on the behavior of IPPs and their physical meanings above-mentioned are applied to biological imaging in this section. In particular, the ex-vivo experiments conducted in this section consist of measuring a non-standardized dissection of a rabbit leg and compare the information obtained from the well-known channels (intensity term $\mathrm{m}_{00}$, retardance $\mathrm{R}, \Delta$ and $\mathrm{P}_{\Delta}$ ) with the results obtained from the new IPP channels.

The MM of the ex-vivo rabbit leg was measured by illuminating the sample with the wavelength of $475 \mathrm{~nm}$. This was done to further inspect surface details of the sample because shorter wavelengths remain more on the surface [22]. The obtained results are demonstrated in Fig. 6. From the 16 components of the Mueller matrix, we identify the intensity 
$\mathrm{m}_{00}$ (Fig. 6(a)), the diattenuation and the polarizance (see Section 2). As in most of the biological samples, diattenuator and polarizance vectors give insignificant and negligible information and for this reason they are not included in Fig. 6 . In Fig. 6(a), the intensity channel reveals an elongated tendon which is basically made of connective tissue. We mark this certain section with a green arrow.

After this first above-shown analysis, the Lu-Chipman decomposition (Eq. 2) is performed to determine the total retardance R (Fig. 6(b)) and the depolarization power $\Delta$ (Fig. 6(b)). We also calculated the degree of polarimetric purity $\mathrm{P}_{\Delta}$ (Fig. 6(d)).
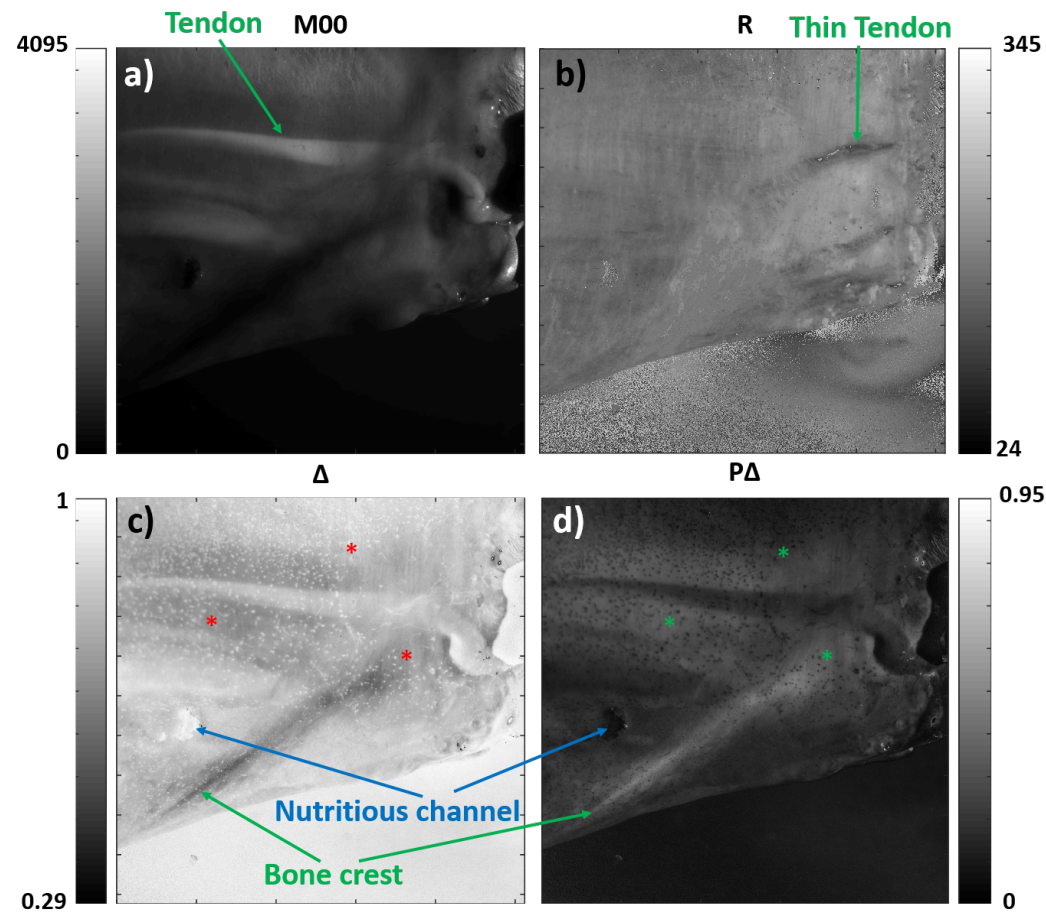

Figure. 6: Polarimetric analysis of a rabbit leg consisting of cortical bone, tendinous tissue and muscle and some spotted structures representing the cortical bone pores $(*)$. (a) regular intensity image (M00); (b) retardance image R; (c) depolarization power $\Delta$ and (d) depolarization index $\mathrm{P}_{\Delta}$.

Total retardance (Fig. 6(b)) presents a thin tendon hidden in the other studied channels. Unfortunately, all the rest parts representing the ex-vivo rabbit leg are indistinguishable in Fig. 6(b). In this case, it is demonstrated that the total retardance measurement is an efficient method if the measured objects show different birefringences. In particular, the tendon is observable because it is an enclosed, wire-like package of a parallel array of collagen fibers which gives a certain degree of birefringence. On the contrary, the other tendons are not observed in this channel because their birefringences are similar to those of the muscle. The similitude can be produced for the changes in the tendon structures near the muscle connections.

Regarding to the two other depolarizing channels, the obtained information is similar but is shown with different contrasts. We can distinguish from Figs. 6(c) and (d) some well-defined structures such as a nutritious channel, a bone crest and some spotted structures hidden in the other channels. We associate the spotted structures (i.e., the dots near the small * points in Figs. 6(c) and (d)) to nutritious foramina. Moreover, we can also observe the same tendon introduced in the intensity channel. For that reason, we can determine that different polarization channels give more information than the intensity-only channel.

Once we have compared the usual polarimetric channels with intensity, the same leg structure is also implemented within the IPP channels. The three IPPs, calculated from the rabbit leg MM by using Eqs. 3 and 4, are presented in Fig. 7. 


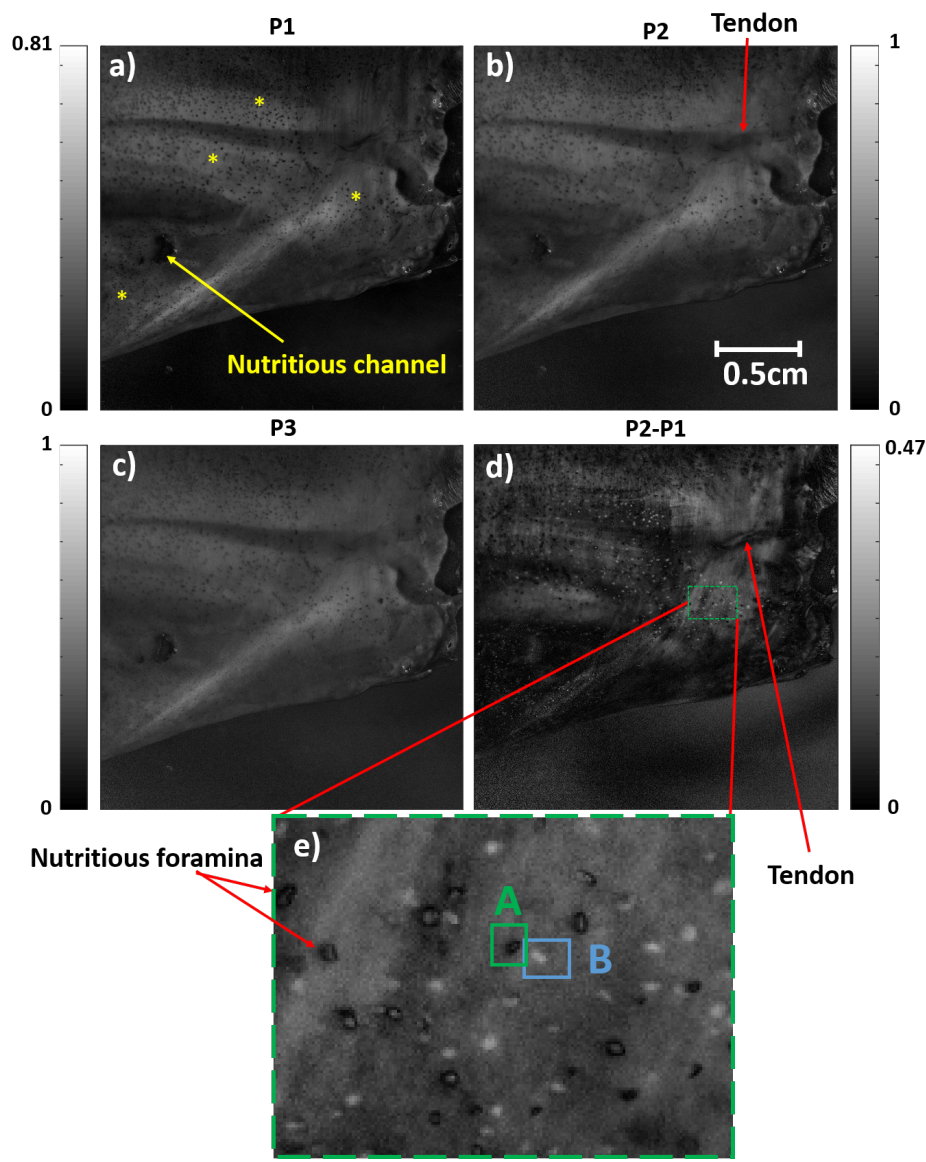

Figure. 7: Polarimetric analysis of a rabbit leg: (a) $\mathrm{P}_{1}$; (b) $\mathrm{P}_{2}$; (c) $\mathrm{P}_{3}$, (d) the difference between $\mathrm{P}_{1}$ and $\mathrm{P}_{2}$; and (e) zoomed image of the ROI marked in (d).

The $\mathrm{P}_{1}$ image is very similar to most used channels, but with a higher contrast between different elements. The depolarization channel $\mathrm{P}_{2}$, on the contrary, has a different response. We are able to identify the thin tendon highlighted in the retardance channel. To further highlight the depolarizing differences, $\mathrm{P}_{2}-\mathrm{P}_{1}$ channel is calculated and thoroughly analyzed (Fig. 7(d)). The subtraction clearly shows the existence of the thin tendon, and also the difference between nutritious foramina. The doted area present in Fig. 7(d) is zoomed in Fig. 7(e) to testify two different nutritious foramina groups. Two particular pieces are presented as dots A and B. In a first approximation, A and B pores are physically different, basically because their depolarization mechanisms are different. Since cortical bone porosity may arise from the distribution of small cortical vessels or the inherent structure of the cortical bone itself along with degenerative or remodelation process, we hypothesize that this white-black variation could be related to the depth of the pore and, thus, to its physiological origin.

Finally, we propose a pseudo-colored image, associating colors to each component, analogously to the three IPP representations in a purity space (Fig. 1), but adding certain weights to increase the importance of some components compared with others. This method is analogous to standard Red-Green-Blue images, but those colours are associated to merit functions depending on the $\mathrm{P}_{1}-\mathrm{P}_{2}-\mathrm{P}_{3}$ channels, and some weights can be associated to the IPPs channels to highlight particular tissues structures. We present two pseudo-colored figures (Figs. 8(a) and (b)) to illustrate the potential of this technique. 


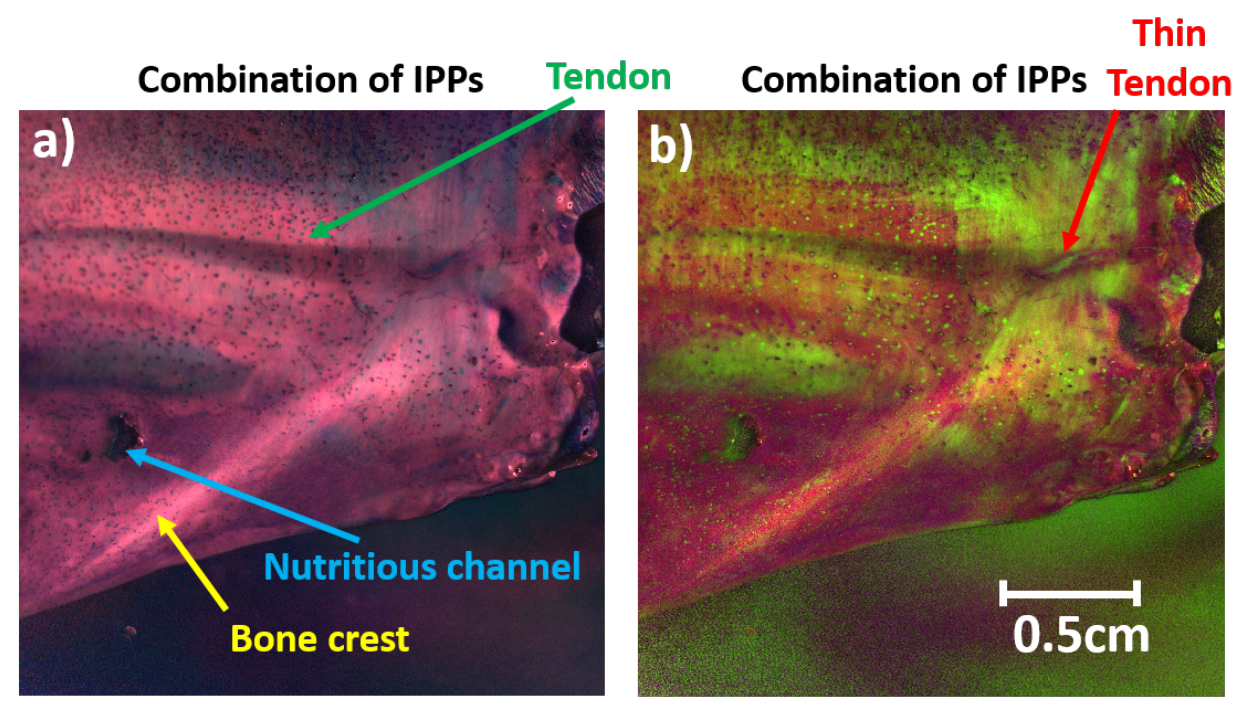

Figure. 8: Pseudo-colored images obtained by using of a non-standardized dissection of a rabbit leg obtained by using different combinations of IPPS channels.

Figure. 8(a) is represented with the intention to increase the visibility of the tendon, nutritious channel, bone crest and the nutritious foramina. Meanwhile, Fig. 8(b) is focused on highlight the thin tendon and the differences between nutritious foramina. More importantly, we want to emphasize that not only the above-mentioned two combinations (i.e., investigated cases represented in Figs. 8(a) and (b)), but also more approaches can be studied to optimize the visualization of particular tissues.

\section{CONCLUSION}

In this paper, we highlight the potential of indices of polarimetric purity as a criterion to characterize and classify depolarizing samples. We also present the synthesis of complex depolarizers from the use of simple polarization components. An example of the potential of this analysis to synthetize materials is discussed with the fabrication of a pure depolarizer. In fact, an acceptable agreement within the IPP between the theoretical ideal depolarizer (sample "E") with a commercial one (sample "F"), is demonstrated.

What is more, we demonstrate as well the interest of using IPPs to extract depolarization information from depolarizing samples, in the context of biological applications. Images obtained by implementing the IPP method provide an enhanced contrast with respect to the conventional channels and they also provide new physical interpretation as it is discussed in this proceeding. Moreover, some hidden structures (in conventional channels) are observable in some IPP channels, this making the technique a powerful structure recognition method. All this situation is provided in the manuscript by studying a particular ex-vivo sample (rabbit leg).

Finally, we introduce a pseudo-colored image method to encode all the depolarization information in one image. The combination formula is not unique and can feature different parts depending on the used formula.

\section{REFERENCES}

[1] R. M. A. Azzam, N. M. Bashara, [Ellipsometry and Polarized Light], North-Holland Publishing Company, Amsterdam, (1977).

[2] J. Hough, Astron. "Polarimetry: a powerful diagnostic tool in astronomy," Geophys 47, 3.31-3.35 (2006).

[3] N. Uribe-Patarroyo, A. Alvarez-Herrero, R. L. Heredero, J. C. del Toro, A. C. López, V. Domingo, J. L. Gasent, L. Jochum, V. Martínez, "You have full-text access to this contentIMaX: a polarimeter based on Liquid Crystal Variable Retarders for an aerospace mission," Phys. Status Solidi C 5, 1041-1045 (2008).

[4] J. S. Tyo, D. L. Goldstein, D. B. Chenault, J. A. Shaw, "Review of passive imaging polarimetry for remote sensing applications,” Appl. Optics 45, 5453-5469 (2006). 
[5] D. M. Shinki, M. T. Ivanov, J. S. Post, S. Vagle, J. T. Cullen, D. K. Hore, “An auto-calibrating Stokes polarimeter for materials characterization," Appl. Optics 51, 4113-4119 (2012).

[6] A. Lizana, M. Foldyna, M. Stchakovsky, B. Georges, N. David, E. GarciaCaurel, "Enhanced sensitivity to dielectric function and thickness of absorbing thin film by combining total internal reflection ellipsometry with standard ellipsometry and reflectometry," J. Phys. D Appl. Phys. 46, 105501 (2013).

[7] T. Yasui, Y. Tohno, T. Araki, "Characterization of collagen orientation in human dermis by two-dimensional second-harmonic-generation polarimetry," J. Biomed. Opt. 9, 259-264 (2004).

[8] L. Graham, Y. Yitzhaky, and I. Abdulhalim, "Classification of skin moles from optical spectropolarimetric images: a pilot study,” J. Biomed. Opt. 18, p.111403 (2013).

[9] Y. Yitzhaky, L. Graham, and I. Abdulhalim, "Analysis of skin moles from optical spectropolarimetric images," Proc. of SPIE 8856, p. 88562J-1 (2013).

[10] T. Novikova, A. Pierangelo, A. De Martino, A. Benali, and P. Validire, "Polarimetric imaging for cancer diagnosis and staging," Opt. Phot. News 23, p. 26-33 (2012).

[11] A. Pierangelo, A. Nazac, A. Benali, P. Validire, H. Cohen, T. Novikova, B.H. Ibrahim, S. Manhas, C. Fallet, M.R. Antonelli, and A. De Martino, "Polarimetric imaging of uterine cervix: a case study," Opt. Express 21, p. $14120-14130(2013)$.

[12]E. Du, H. He, N. Zeng, M. Sun, Y. Guo, J. Wu, S. Liu and H. Ma, "Mueller matrix polarimetry for differentiating characteristic features of cancerous tissues," J. Biomed. Opt. 19, 076013 (2014).

[13] A. Lizana, A. Van Eeckhout, K. Adamczyk, C. Rodríguez, J. C. Escalera, E. Garcia-Caurel, I. Moreno, J. Campos, "Polarization gating based on Mueller matrices," J. Biomed. Opt. 22, 056004 (2017).

[14]D. Goldstein, [Polarized Light], 2nd ed., Marcel Dekker, New York (2003).

[15]E. Garcia-Caurel, R. Ossikovski, M. Foldyna, A. Pierangelo, B. Drévillon, A. De Martino, [Advanced Mueller Ellipsometry Instrumentation and Data Analysis] Eds: M. Losurdo, K. Hingerls, Springer-Varlag, Berlin (2013).

[16]R. A. Chipman, [Polarimetry: Handbook of Optics], 2nd ed. McGrawHill, New York, (1995).

[17] J. J. Gil, R. Ossikovski, [Polarized Light and the Mueller Matrix Approach] CRC Press, Boca Raton, FL (2016).

[18]J. J. Gil, J. M. Correas, P. A. Melero, C. Ferreira, "Generalized polarization algebra,” Monog. Sem. Mat. G. Galdeano 31, 161 (2004).

[19] J. J. Gil, "Invariant quantities of a Mueller matrix under rotation and retarder transformations," J. Opt. Soc. Am. A 33, 52 (2016).

[20] A. Peinado, A. Lizana, J. Vidal, C. Iemmi, and J. Campos, “Optimization and performance criteria of a Stokes polarimeter based on two variable retarders," Opt. Express 18, 9815-9830 (2010).

[21] R. Ossikovski and K. Hingerl, "General formalism for partial spatial coherence in reflection Mueller matrix polarimetry,” Opt. Lett. 41, 4044-4047 (2016).

[22]F. H. Mustafa, M. S. Jaafar, "Comparison of wavelength-dependent penetration depths of lasers in different types of skin in photodynamic therapy," Indian J. Phys. 87, 203 (2013).

[23] J. J. Gil, "Structure of polarimetric purity of a Mueller matrix and sources of depolarization," Opt. Commun. 368, 165-173 (2016).

\section{ACKNOWLEDGEMENTS}

We acknowledge the financial support of Spanish MINECO (FIS2015-66328-C3-1-R, FIS2015-66328-C3-3-R and fondos FEDER); Catalan Government (SGR 2014-1639). 